\title{
Concepções e práticas dos enfermeiros da estratégia saúde da família acerca da violência infantil
}

RESUMO | Objetivo: Conhecer as concepções e práticas dos enfermeiros da Estratégia Saúde da Família (ESF) acerca dos casos de violência infantil. Método: Estudo descritivo, com abordagem qualitativa, realizado com enfermeiros de unidades de ESF em um município do estado de São Paulo, Brasil, no ano de 2017. Para coleta de dados, utilizou-se a entrevista semiestruturada, empregouse a técnica de análise temática e COREQ para redação cientifica. Aprovado pelo CEP sob CAAE 65991417.0.0000.5431. Resultados: Da análise, emergiram como categorias: conhecimento e percepção acerca da violência infantil, posicionamento e ações desenvolvidas frente à violência infantil nas unidades e aspectos éticos e responsabilidades do profissional enfermeiro. Conclusões: Os discursos revelaram que as profissionais obtêm conhecimento sobre a temática e conseguem identificar os sinais e sintomas de violência nas crianças e adolescentes. Apesar disso, as enfermeiras manifestaram insegurança no processo de notificação, demonstrando medo para realizar e se envolver legalmente frente aos casos.

Descritores: Criança; Violência Doméstica; Saúde Pública; Enfermagem.

\begin{abstract}
Objective: To know the conceptions and practices of nurses in the Family Health Strategy (ESF) about cases of child violence. Method: Descriptive study with a qualitative approach, carried out with nurses from FHS units in a city in the state of São Paulo, Brazil, in 2017. For data collection, a semi-structured interview was used, using the technique of thematic analysis and COREQ for scientific writing. Approved by CEP under CAAE 65991417.0.0000.5431. Results: From the analysis, the following categories emerged: knowledge and perception about child violence, positioning and actions taken in relation to child violence in the units, and ethical aspects and responsibilities of the professional nurse. Conclusions: The speeches revealed that the professionals obtain knowledge on the subject and are able to identify the signs and symptoms of violence in children and adolescents. Despite this, the nurses expressed insecurity in the notification process, showing fear to perform and get legally involved in the cases
\end{abstract}

Descriptors: Child; Domestic Violence; Public Health; Nursing.

\section{RESUMEN | Resumen}

Objetivo: Conocer las concepciones y prácticas de los enfermeros en la Estrategia de Salud de la Familia (ESF) sobre los casos de violencia infantil. Método: Estudio descriptivo con abordaje cualitativo, realizado con enfermeros de unidades de la ESF de una ciudad del estado de São Paulo, Brasil, en 2017. Para la recolección de datos se utilizó una entrevista semiestructurada, utilizando la técnica de análisis temático y COREQ para escritura científica. Aprobado por CEP bajo CAAE 65991417.0.0000.5431. Resultados: Del análisis surgieron las siguientes categorías: conocimiento y percepción sobre la violencia infantil, posicionamiento y acciones tomadas en relación a la violencia infantil en las unidades, y aspectos éticos y responsabilidades del profesional de enfermería. Conclusiones: Los discursos revelaron que los profesionales obtienen conocimientos sobre el tema y son capaces de identificar los signos y síntomas de violencia en niños y adolescentes. Pese a ello, las enfermeras expresaron inseguridad en el proceso de notificación, mostrando miedo a desempeñarse e involucrarse legalmente en los casos.

Descriptores: Envejecimiento; Anciano; Depresión; Hogares para ancianos; Enfermería.

\section{Adriana Aparecida Moreira de Paula}

Enfermeira. Bacharel em Enfermagem pela Escola Superior do Cruzeiro (ESC), CruzeirO, SP.

ORCID: 0000-0001-9398-2944

\section{Maria Creusa Moreira}

Enfermeira. Bacharel em Enfermagem pela Escola Superior do Cruzeiro (ESC), Cruzeiro, SP.

ORCID: 0000-0002-0688-7920

\section{Hercules de Oliveira Carmo}

Enfermeiro. Docente em Enfermagem da Escola Superior do Cruzeiro (ESC). Doutorando em Gerenciamento em Enfermagem pela Escola de Enfermagem da Universidade de São Paulo (EEUSP).

ORCID: 0000-0002-6996-4233

\section{Silvia Maria de Carvalho Farias}

Enfermeira. Docente e Coordenadora do Curso de Graduação em Enfermagem da Escola Superior do Cruzeiro (ESC). Mestra em Unidade de Terapia Intensiva pela Sociedade Brasileira de Terapia Intensiva (SOBRATI/IBRATI).

ORCID: 0000-0002-0318-2810

\section{Leonardo dos Santos Moreira}

Enfermeiro. Docente em Enfermagem da Faculdade Anhanguera Campinas (FAC). Especialista em Enfermagem Obstétrica e Ginecológica (FEHIAE) - Instituto Israelita de Ensino e Pesquisa Albert Einstein - SP. ORCID: 0000-0001-8689-1171

Received: 27/08/2021

Approved: 30/11/2021
INTRODUÇÃO

A violência "o desafio do século", é um fenômeno complexo, está difundida em todo o tecido social e identificada internacionalmente como um grave problema de Saúde Pública e de Direitos Humanos.

A violência pode ser considerada como uso da força física ou do poder, real ou em ameaça, contra si próprio, contra outra pessoa, ou contra um grupo ou uma comunidade, que resulte ou tenha qualquer possibilidade de resultar em lesão, morte, dano psicológico, 
deficiência de desenvolvimento ou privação (1).

Existem diversas formas de violência e com diferentes pessoas figurando no polo ativo e no passivo da prática delituosa. A Organização Mundial de Saúde (OMS) define a violência como todas as formas de maus-tratos emocionais, físicas, abuso sexual, negligência, ou outras formas de exploração, com possibilidade de resultar em danos potenciais ou reais à saúde das crianças, sobrevivência, desenvolvimento ou dignidade no contexto de uma relação de responsabilidade, confiança ou poder $(1,2)$.

No Brasil, o despertar e reconhecimento para a necessidade de proteção integral à criança e ao adolescente, veio a partir da publicação da Lei n. 8.069 no ano de 1990, que dispõe sobre o Estatuto da Criança e do Adolescente - ECA (3) enfatizando que, nenhuma criança ou adolescente deve ser objeto de qualquer forma de negligência, discriminação, exploração, violência, crueldade e opressão, e tal será punido na forma da lei a qualquer atentado (4).

O ministério da Saúde apresentou que no ano de 2017 foram registrados 126.230 casos de violência contra crianças e adolescentes, correspondendo assim a, 42\% do total de casos notificados no mesmo ano. Houve registros de 21.559 mortes por causas externas, acidentes e violência com crianças e adolescentes (5). Os efeitos da violência sobre as vidas daqueles que sobrevivem são imensuráveis, repercutindo em problemas emocionais, físicos, sociais e econômicos, e estes, contribuindo para um ciclo vicioso de violência que se perpetua (6).

A Organização das Nações Unidas (ONU) fez um alerta para o aumento de violência doméstica que pode ocorrer em meio à quarentena imposta em resposta à pandemia (7) e, convocou os governos e a comunidade internacional para proteger as crianças destes riscos, por meio de uma resposta coletiva, incluindo saúde mental e apoio psicossocial, proteção social e cuidado para as mais vulneráveis e que estão instituições de acolhimento (8).

\section{ministério da Saúde apresentou que no ano de 2017 foram registrados 126.230 casos de violência} contra crianças e adolescentes, correspondendo assim a, $42 \%$ do total de casos notificados no mesmo ano. Houve registros de 21.559 mortes por causas externas, acidentes e violência com crianças e adolescentes

No que tange a complexidade da violência doméstica contra crianças e adolescentes, faz-se necessário desenvolver um olhar multiprofissional e ações intersetoriais que melhorem as condições de vida destas vítimas. Os profissionais de saúde podem contribuir para a mudança do cenário do fenômeno da violência, assumindo a responsabilidade legal perante a notificação dos casos e a assistência às crianças e adolescentes (9).

Nas últimas décadas, percebe-se uma crescente participação dos enfermeiros em diferentes espaços de promoção, proteção e recuperação da saúde de crianças e adolescentes, tais como nas escolas e nas Unidades de Estratégia de Saúde da Família - ESF (10). Sendo assim, torna-se extremamente importante que estes profissionais estejam preparados para o enfrentamento e a prevenção da violência contra crianças e adolescentes.

Diante destes apontamentos, este estudo teve como objetivo: Conhecer as concepções e práticas dos enfermeiros da Estratégia Saúde da Família acerca dos casos de violência infantil.

\section{MÉTODO}

Trata-se de um estudo exploratório, descritivo, com abordagem qualitativa, desenvolvido em cinco unidades de ESF de uma cidade no interior do estado de São Paulo, Brasil.

Participaram cinco enfermeiras que atuam nos serviços. A escolha desta categoria profissional deu-se pela proximidade com a população em estudo e pela dinâmica do processo de trabalho em ESF, tendo o enfermeiro mediador do serviço. Assim, adotaram-se os seguintes critérios de inclusão: ser enfermeiro cadastrado na área de abrangência das unidades de ESF, atuar por um período mínimo de seis meses e estar desempenhando suas atividades profissionais durante o período de coleta de dados. E, como critério de exclusão: estar ausente do trabalho por motivo de férias, afastamentos ou licenças no período da coleta dos dados.

A coleta de dados foi realizada entre os meses de abril a maio de 2018, 
por meio de entrevistas semiestruturadas, gravadas em mídia digital e, tiveram em média 25 minutos de duração. O local escolhido para sua realização foram as próprias unidades ESF.

Para manter o rigor no estudo foram utilizados critérios estabelecidos para o Reporting Pesquisa Qualitativa COREQ como ferramenta de protocolo para redação cientifica (11). Todavia, após as transcrições das entrevistas, não houve retorno aos participantes para comentar o assunto.

O instrumento para coleta de dados baseou-se em um roteiro composto pelas seguintes questões norteadoras: O que é violência e quais são as suas percepções acerca da violência infantil? Quais ações que você enquanto enfermeiro desta unidade de ESF tem desenvolvido no enfrentamento da violência infantil? Com qual rede de apoio e proteção a sua unidade de ESF dispõe para este enfrentamento?.

Após a coleta, a análise foi gerada a partir da exploração do material, realizou-se a codificação por nomes de pedras preciosas (Diamante, Ametista, Rubi, Esmeralda e Topázio) e caracteres alfanuméricos (E1, E2, E3, E4 e E5) das participantes a fim de manter o sigilo e anonimato, e posteriormente, seguiu-se com a classificação das unidades temáticas.

A unidade temática corresponde a uma transformação dos dados brutos, que por meio do recorte, agregação e enumeração permitem definir as unidades de significado, as quais conduzem para uma descrição exata das características pertinentes do conteúdo, culminando com a categorização (12).

Atendendo à resolução n. 466/2012 do Ministério da Saúde (13), que regulamenta a realização de pesquisas envolvendo seres humanos, o projeto desta pesquisa foi aprovado pelo Comitê de Ética em Pesquisa pelo protocolo CAAE 65991417.0.0000.5431 e sob o parecer $n^{\circ} 1.985 .010$, em 27 de março de 2017.

\section{RESULTADOS}

A caracterização das participantes demonstrou que, a média de idade foi de 35,4 anos e o tempo de atuação nas unidades ESF de 6,8 anos. Quanto à formação específica, (3) enfermeiras tinham especialização em Saúde da Família e (4) revelaram ter capacitação

\section{Os relatos das profissionais denotam que a apresentação dos sinais, sintomas e comportamentos nas crianças e adolescentes são os fatores mais citados como forma de (re)conhecer a violência.}

sobre casos de violência contra criança e adolescente.

Ao final do processo de análise do material coletado, emergiram as seguintes categorias temáticas: conhecimento e percepção acerca da violência infantil, posicionamento e ações desenvolvidas frente à violência infantil nas unidades e aspectos éticos e responsabilidades do profissional enfermeiro.

Conhecimento e percepção acerca da violência infantil

Nessa categoria, foi investigada a concepção construída sobre o termo 'violência infantil' e, a percepção das enfermeiras que atuavam nas unidades de ESF.

Os relatos das profissionais denotam que a apresentação dos sinais, sintomas e comportamentos nas crianças e adolescentes são os fatores mais citados como forma de (re)conhecer a violência.

Suspeito quando a sinais de violência, através do comportamento da criança. Pode apresentar gestos que alguma coisa não está bem, olhar de tristeza, medo, receio; observo o perfil da família; como a mãe trata essa criança, muitas vezes, os pais nem deixam a criança a falar (Diamante - E1).

A violência interpõe-se como uma poderosa ameaça ao direito à vida e a saúde da criança e de sua família. A exposição da criança a qualquer forma de violência seja de natureza física, sexual e psicológica, assim como a negligência e abandono principalmente na fase inicial da sua vida, poderá comprometer sua vida como um todo. (Esmeralda - E2).

As características são variáveis de criança para criança, de caso para caso; no caso que tive conhecimento: à criança estava hiperativa, agitada, com a necessidade de chamar a atenção de todos ao seu redor. (Rubi - E3).

Eu percebi durante um dia de calor, no qual a criança estava toda coberta; a criança estava muito falante e o adulto responsável não a deixava sozinha com o profissional de saúde (Topázio - E4).

Identifico a partir que vejo a 
criança assustada, com medo e receio de falar e com sinais físicos aparentes de violência (Ametista - E5)

Os enfermeiros nas unidades de ESF estão constantemente em contato com crianças, adolescentes e seus familiares, e assim, conseguem aplicar as suas habilidades e competências técnicas na identificação dos possíveis casos de violência intrafamiliar.

Diante deste contexto, as profissionais foram abordadas quanto à atuação perante os casos suspeitos e/ou confirmados de violência infantil, configurando assim, a outra categoria.

Posicionamento do enfermeiro frente à violência infantil

Neste núcleo temático, as participantes da pesquisa expuseram as suas experiências e atuação frente aos casos de violência contra a criança e adolescente.

A princípio peço ao agente comunitário que realize um maior número de visitas nesse lar, peço que observem a criança ainda melhor. E caso, o profissional [agente comunitário] verifique uma forte suspeita ou tenha certeza do ocorrido, peço que me relate onde, e eu aciono o Conselho Tutelar e a Vigilância. (Diamante $-\mathrm{E} 1)$

Geralmente tentamos [equipe] conversar [criança] e com a família, observar a criança, comunicar o Conselho Tutelar e fazer visitas ao lar desta criança, envolvendo todos os profissionais. (Esmeralda $-\mathrm{E} 2)$

Quando chega ao nosso conhecimento, fazemos o acompanhamento multidisciplinar para família, cada profissional dentro de suas competências e área de atuação, com sigilo e ética. (Rubi - E3)

Oriento o responsável para observar mudanças bruscas no comportamento da criança, pois, de alguma forma, ela irá chamar atenção de quem cuida dela. Se confirmada à violência, devo

A partir dos relatos, percebe-se que a abordagem colaborativa e interprofissional tem facilitado a identificação de sinais ou comportamentos de violência e o manejo do atendimento perante os casos.

comunicar as autoridades competentes e oferecer os exames de teste rápido: HIV I e II, VDRL, Hepatite B e C (Topázio - E4).

Solicito apoio e investigação do Conselho Tutelar (Ametista - E5).

A partir dos relatos, percebe-se que a abordagem colaborativa e interprofissional tem facilitado a identificação de sinais ou comportamentos de violência e o manejo do atendimento perante os casos.

De fato, o trabalho em equipe é o pilar da qualidade da assistência nas unidades de ESF. No entanto, verifica-se que algumas enfermeiras carecem de melhores entendimentos sobre a identificação dos possíveis agressores, sendo muitas das vezes, o próprio responsável e/ou familiar e das condutas que devem ser tomadas.

Aspectos éticos e as responsabilidades do profissional enfermeiro

Nessa categoria, foram abordados os aspectos éticos e as responsabilidades das enfermeiras frente aos casos suspeitos e/ou confirmados de violência contra a criança e adolescente. Conforme os relatos:

Realizo as notificações muitas das vezes com medo, pois é uma situação delicada, envolvendo denuncia e pessoas, mas temos nossa identidade preservada e podemos prosseguir com nosso trabalho (Diamante - E1).

A partir do respaldo legal que temos, podemos desenvolver um trabalho mais eficaz com essas vítimas (Esmeralda - E2).

Sabemos do respaldo legal, das ações do Conselho Tutelar, das leis vigentes para as vítimas de violência, mas depende da família dar seguimento na maioria das vezes (Rubi - E3).

Quando observo alguma coisa diferente nessas crianças, entramos em acordo 'médico e enfermeira' para realizarmos as condutas necessárias em cada caso (Topázio - E4).

A atuação frente aos casos depende muito do comprometimento e 
da força de vontade de todos os profissionais (Ametista - E5).

Os relatos revelam que as enfermeiras têm dificuldades para operacionalizar a notificação, e, demonstram medo para o realizar e se envolver legalmente frente aos casos.

\section{DISCUSSÃO}

O presente estudo apontou as concepções e práticas assistenciais das enfermeiras de unidades ESF acerca fenômeno violência infantil.

Os discursos revelaram que as profissionais obtêm conhecimento sobre a temática e principalmente, conseguem identificar os sinais e sintomas de violência nas crianças e adolescentes. Outro dado relevante apontado refere-se, ao fato da proximidade com os sujeitos e a própria dinâmica do programa ESF, ampliando assim, as possibilidades de investigação, identificação e conduta pelas profissionais. Apesar disso, as enfermeiras manifestaram insegurança no processo de notificação, e, demonstraram medo para realizar e se envolver legalmente frente aos casos.

A violência é um agravo de grande relevância mundial, assim o monitoramento e a análise tornam-se de extremamente importância. Principalmente, em epidemiologia, o conhecimento sobre os casos permite dimensionar a magnitude do problema e possibilitam definir medidas efetivas para intervenção, de acordo com o perfil da população acometida (14).

No ano de 2019, foram registradas 159.063 denúncias de maus-tratos pelo disque Direitos Humanos (Disque 100), revelando-se, um aumento de 15\% em relação ao ano anterior. Dessas indicações, 86.837 eram de violências contra crianças e adolescentes (55\%), sendo: $38 \%$ relativas à negligência, $23 \%$ à violência psicológica, $21 \%$ à violência física, $11 \%$ à violência sexual, 3\% à exploração/ao trabalho infantil e 3\% associadas a outros agravos violentos. O local mais frequente das ocorrências foi à casa da vítima (15).

Já em 2020, com a pandemia de COVID-19, o impacto da violência contra crianças e adolescentes foram ainda maiores. O Painel de Dados da Ouvidoria Nacional dos Direitos Humanos revelou que ocorreram 95.247 denúncias e 368.333 violações, sendo assim, o grupo vulnerável com maior número de denúncias relatadas no país (16).

Os indícios de violência mais percebidos nos atendimentos dos profissionais de saúde da atenção básica do munícipio de Belém no estado do Pará foram os relacionados, aos sinais físicos no corpo da vítima $(46,69 \%)$, com destaque para os hematomas $(28,30 \%)$, e os sinais comportamentais $(28,19 \%)$, evidenciando-se o comportamento agressivo ou retraído (17).

A elevada proporção de recorrência da violência na infância é preocupante, aponta as vulnerabilidades sociais a que essas crianças e suas famílias estão expostas, e também, as medidas que deveriam ser tomadas para protegê-las (18). Entretanto, a literatura ressalta que violência contra a criança e adolescente é difícil de ser desvenda$\mathrm{da}$, principalmente pelo fato de muitas das vezes ocorrer na esfera privada e doméstica, ser resguardada pelo silêncio e medo, pela impunidade de seus agentes e pelas distintas formas e classificações (19).

Sabe-se que os serviços de saúde da Atenção Primária à Saúde (APS), principalmente as unidades de ESF, tem grande potencial no que diz respeito ao enfrentamento da violência. As ações desenvolvidas nas ESF são direcionadas para intervenções de saúde com centralidade na família e abordagens comunitárias. Estas possibilidades podem ampliar o diálogo, mapear os riscos, criar e implementar protocolos de vigilância e de monitoramento da violência.
Entretanto, algumas pesquisas têm apresentado que os profissionais das unidades de ESF encontram-se despreparados para lidar com esta questão acontecem nos seus territórios (20-22). O estudo (23) revelou que na prática, os profissionais de enfermagem demonstram medo de notificar os casos de violência intrafamiliar, delegando esta função a outros profissionais, e esse tipo de comportamento, está atrelado com o medo dos profissionais de saúde de se expor ao agressor, pois sentem-se desprotegidos e desamparados $(23,24)$. Ambos apresentam similaridades, aos desafios apontados pelas enfermeiras entrevistas neste estudo, referindo-se ao medo, à insegurança e às fragilidades da rede de apoio e que necessitam ser superados.

O medo de envolvimento legal nos casos, as precariedades na formação acadêmica e na rede de apoio do município, assim como a ausência de proteção aos profissionais envolvidos no acompanhamento dos casos são alguns exemplos que envolvem o baixo índice de notificação dos casos de violência contra crianças em todo o país (25).

A detecção precoce é fundamental e faz-se necessária para a eficácia da intervenção e quebra do ciclo de violência. A pesquisa (26) realizada com profissionais de nível superior que atuavam nas unidades ESF, identificou que, eles possuíam dificuldades para identificar os sinais de abuso e violência, não sabiam como realizar a denúncia e necessitavam de educação continuada sobre o assunto. Outras investigações constataram que, os profissionais da ESF fazem maior referência às manifestações físicas da violência, não mencionando situações de violência psicológica (20) e afirmaram notificar apenas casos de violência física e sexual, demonstrando como dificilmente são reportadas suas manifestações menos explícitas (27).

À vista disso, é importante que os profissionais que podem detectar os si- 
nais de abusos e de violências, como aqueles que trabalham no âmbito da Saúde e da Educação, estejam capacitados para lidar com essa problemática no cotidiano, possibilitando a efetivação dos direitos e o acolhimento das vítimas (28).

Diante de todo contexto, é imprescindível que os enfermeiros estejam qualificados e preparados para o manejo clínico e psicológico dos casos, e tenham pleno o conhecimento da legislação que asseguram estes direitos. Compete ao enfermeiro, atuar na prevenção da violência doméstica infantil, diagnosticar os riscos nos territórios de atuação e, quando identificar e diagnosticar, realizar a denúncia em tempo hábil, para que possa garantir a integridade física e emocional destas crianças e adolescentes.

\section{CONCLUSÃO}

Os resultados do presente estudo permitiram a identificação que as enfermeiras entrevistas têm conhecimento sobre a temática de violência contra a criança e adolescente, e principalmente, conseguem identificar os sinais e sintomas para os casos suspeitos e/ou confirmados. Entretanto, as enfermeiras manifestaram insegurança no processo de notificação, e, demonstraram medo para realizar e se envolver legalmente frente aos casos. Soma-se a isso a necessidade do entendimento da notificação da violência, como relevante instrumento para o monitoramento, o enfrentamento e a construção de políticas públicas.

Diante disso, faz se necessário pen- sar na ampliação de práticas educativas para melhor conhecimento e abordagem, bem como reformular ações da rede de proteção dos profissionais e das vítimas, pois isto, tem influenciado o trabalho prático das enfermeiras, refletindo principalmente no número de notificações e da condução dos casos.

Defende-se que, mesmo com as limitações referentes ao tamanho da amostra e tipo de abordagem metodológica, os resultados apontam para a necessidade da realização de formação continuada direcionada. E por fim, sugere-se também, ampliar as discussões com outros órgãos competentes a fim de organizar uma rede de atenção e proteção a estas crianças e adolescentes e aos profissionais envolvidos do município estudado.

\section{Referências}

1. World Health Organization (WHO). World report on violence and health. Geneva: WHO; 2002.

2. World Health Organization (WHO). Preventing child maltreatment: a guide to taking action and generating evidence. Geneva: World Health Organization; 2006.

3. Brasil. Lei $n^{\circ} .8069$ de 13 de julho de 1990 - Estatuto de Criança e do Adolescente. Brasília: Congresso Nacional, 1990.

4. Digiácomo MJ, de Amorim Digiácomo I. Estatuto da criança e do adolescente anotado e interpretado. Ministério Público do Estado do Paraná. Centro de Apoio Operacional das Promotorias da Criança e do Adolescente, 2017.

5. Brasil. Ministério da Saúde. DATASUS: Tecnologia da Informação a Serviço do SUS. Indicadores de saúde: causas externas [Internet]. Brasília, DF: Ministério da Saúde; 2017. Disponível em: http://tabnet.datasus.gov.br/ cgi/tabcgi.exe?sim/cnv/ext10uf.def

6. Almeida MGB. A violência na sociedade contemporânea. Porto Alegre: EDIPUCRS; 2010.

7. Organização das Nações Unidas. Chefe da ONU alerta para aumento da violência doméstica em meio à pandemia do coronavírus. Disponível em: https://nacoesunidas.org/chefe-da-onu-aler ta-para-aumento-da-violencia-domestica-em-meio-a-pandemia-do-coronavirus/

8. World Health Organization (WHO). Global status report on preventing violence against children 2020. (2020). Disponível em: https://violenceagainstchildren.un.org/sites/violenceagainstchildren.un.org/files/2020/ global_status_report/global_status_report-eng.pdf

9. Leite JT, Beserra MA, Scatena L, Silva LMPD, Ferriani MDGC. Enfrentamento da violência doméstica contra crianças e adolescentes na pers- pectiva de enfermeiros da atenção básica. Rev. Gaúcha Enferm. 2016 37(2): e55796.

10. Batista JMS, Trigueiro TH, Lenardet MH, Mazza VA, Labronice LM. 0 modelo bioecológico: desvendando contribuições para a práxis da enfermagem diante da violência doméstica. Esc Anna Nery. 2013; 17(1):173-8 Disponível em: http://www.scielo.br/scielo.php?script=sci_arttext\&pi$\mathrm{d}=$ S1414-81452013000100024

11. Tong A, Flemming $K$, Mclnnes E, Oliver S, Craig J. Enhancing transparency in reporting the synthesis of qualitative research: ENTREQ. BMC Med Res Methodol. 2012;12:181-8.

12. Minayo MCS. 0 desafio do conhecimento: pesquisa qualitativa em saúde. São Paulo, SP: Hucitec. 2014; 21-37.

13. Brasil. Ministério da Saúde. Conselho Nacional de Saúde. Comissão Nacional de Ética em Pesquisa (CONEP). Resolução nº 466, de 2012. Brasília; 2012.

14. da Silva Oliveira Y, Rodrigues da Silva D, Mundim Pombo AP, Ferreira Moura R. Epidemiologia da violência sexual infanto-juvenil no município de São Paulo. Nursing (São Paulo), 2020; 23(271): 5055-66.

15. Brasil. Ministério da Mulher, da Família e dos Direitos Humanos [Internet]. Ouvidoria Nacional de Direitos Humanos (ONDH). Disque 100. Relatório violência contra crianças e adolescentes. Brasília, DF: ONDH; 2019. Disponível em: https://www.gov.br/mdh/pt-br/acesso-a-informacao/disque-100-1

16. Brasil. Ministério da Família da Mulher e dos Direitos Humanos [Internet]. Painel de Dados da Ouvidoria Nacional de Direitos Humanos. 2020. Disponível em: https://www.gov.br/mdh/pt-br/ondh/paineldedadosdaon$\mathrm{dh}$ 


\section{Referências}

17. Veloso MMX, Magalhães CMC, Cabral IR. Identificação e notificação de violência contra crianças e adolescentes: limites e possibilidades de atuação de profissionais de saúde. Mudanças-Psicologia da Saúde. 2017; 25(1): 1-8. Disponível em: https://www.metodista.br/revistas/revistasmetodista/index.php/MUD/article/view/7047

18. Farias MS, Souza CS, Carneseca EC, Passos ADC, Vieira EM. Caracterização das notificações de violência em crianças no município de Ribeirão Preto, São Paulo, no período 2006-2008. Epidemiol Serv Saude. 2016; 25(4):799-806. DOI: http://dx.doi.org/10.5123/s167949742016000400013

19. Sociedade de Pediatria de São Paulo. Sociedade Brasileira de Pediatria. Manual de atendimento às crianças e adolescentes vítimas de violência. 2nd ed. Brasília, DF: CFM; 2018.

20. Costa DKG, Reichert LP, França JRFS, Collet N, Reichert APS. Concepções e práticas dos profissionais de saúde acerca da violência intrafamiliar contra crianças e adolescentes. Trab. Educ. Saúde. 2015; 13(supl. 2): 79-95.

21. Trabbold VLM, Caleiro RCL, Cunha CF, Guerra AMC. Concepções sobre adolescentes em situação de violência sexual. Psicologia \& Sociedade. 2016; 28(1): 74-83.

22. Oliveira NFD, Moraes CLD, Junger WL, Reichenheim ME. Violência contra crianças e adolescentes em Manaus, Amazonas: estudo descritivo dos casos e análise da completude das fichas de notificação, 2009-2016. Epidemiologia e Serviços de Saúde. 2020; 29: e2018438. Disponível em: https://www.scielosp.org/article/ress/2020.v29n1/e2018438/
23. Lima MCCS, Costa MCO, Bigras M, Santana MAO, Alves TDB, Nascimento $\mathrm{OC}$, et al. Atuação profissional da atenção básica de saúde face à identificação e notificação da violência infanto-juvenil. Rev Baiana Saúde Pública. 2011; 35 (1): 118-37. DOl: https://doi.org/10.22278/23182660.2011.v35.n0.a151

24. Al-Ali MN, Lazenbatt A. A cross-cultural comparative study of undergraduate health care professional students'knowledge, definitions, education, and training experience of domestic violence in Northern Ireland and Jordan. SAGE Open 2012; 2(4):1-11. Disponível em: https://journals. sagepub.com/doi/full/10.1177/2158244012464974

25. Moreira GAR, Vieira LJES, Deslandes SF, Pordeus MAJ, Gama IS, BriIhante AVM. Fatores associados à notificação de maus-tratos em crianças e adolescentes na atenção básica. Ciência \& Saúde Coletiva. 2014; 19(10):4267-4276 .

26. Martins-Júnior PA, Ribeiro DC, Peruch GSDO, Paiva SM, Marques LS, Ramos-Jorge ML. Abuso físico de crianças e adolescentes: os profissionais de saúde percebem e denunciam?. Ciência \& Saúde Coletiva. 2019; 24: 2609-2616. Disponível em: https://www.scielosp.org/article/csc/2019. v24n7/2609-2616/

27. Oliveira SM et al. Notificação de violência contra crianças e adolescentes: atuação de enfermeiros de unidades básicas. Revista Enfermagem Uerj. 2013; 21(1): 594-599.

28. Ferreira CLS, Côrtes MCJW, Gontijo ED. Promoção dos direitos da criança e prevenção de maus tratos infantis. Ciência \& Saúde Coletiva. 2019; 24: 3997-4008. Disponível em: https://www.scielosp.org/article/ csc/2019.v24n11/3997-4008/pt/ 